\title{
Erratum: Nonlocal gravity and structure in the Universe [Phys. Rev. D 90, 043535 (2014)]
}

\author{
Scott Dodelson and Sohyun Park
}

(2) (Received 10 July 2018; published 25 July 2018)

DOI: 10.1103/PhysRevD.98.029904

The mathematical expressions written in Phys. Rev. D 90, 043535 (2014) were all correct. However, there was a mistake in the numerical code, which affected the graphs and led to wrong conclusions. The correction of the graphs and further analysis can be found in Phys. Rev. D 97, 044006 (2018). 